age, SES, number of children, smoking, alcohol and body mass index.

Results 2057 incident cases of breast cancer were identified. Compared to day work, self-reported shift work without nights at baseline was associated with an increased risk of breast cancer during a follow-up period of less than 10 year, after adjustment (HR 1.33, 95\% CI 1.15-1.55). In contrast, follow-up for $>=10$ years showed no increased HR. Night shift work was most notably (HR 2.05, 95\% 1.04-4.01) associated with breast cancer among employees 50 years or older after 10 years of follow-up. The risk of breast cancer tended to depend on earlier exposure time. The results of the more recent payroll sub-cohort showed no association of shift work and breast cancer, including intensity and duration of night shift work.

Conclusion This study gives some indications of an increased risk of breast cancer among subgroups of shift-working Finnish public sector employees. However, insufficient information on past exposure to, and intensity of night work, limits the ability to draw firm conclusions.

\section{0-225 EXPLORING THE IMPACT OF NIGHT SHIFT WORK AND MELATONIN ON METHYLATION IN CIRCADIAN GENES}

'Jennifer Ritonja, Parveen Bhatti, Lisa Flaten, Danai G Topouza, Qing Ling Duan, Michael Leung, Francine Durocher, Joan E Tranmer, Kristan J Aronson. 'Queen's University, Canada

\subsection{6/OEM-2021-EPI.141}

Introduction Night shift work is associated with increased cancer risk, but the molecular mechanisms are not well-understood. It is hypothesized that melatonin suppression due to night shift work could impact DNA methylation in circadian genes, although this has been evaluated by few studies.

Objectives This study explored the relationship between night shift work parameters and patterns of melatonin secretion on methylation in circadian genes among women.

Methods A cross-sectional study was conducted in 2019-2020 among 74 female healthcare employees who participated in a previous study in which urinary melatonin levels were evaluated over a 48-hour period. Participants provided information on demographics, lifestyle behaviors, and night shift work such as current night shift work pattern, duration in years, and intensity (consecutive nights). The Illumina Infinium MethylationEPIC beadchip was applied to DNA extracted from new blood samples to measure methylation at 1150 CpG loci across 22 circadian genes. Multiple linear regression was used to examine the association between night shift work, melatonin parameters and methylation levels at each $\mathrm{CpG}$ site, while accounting for the false-discovery rate $(q=0.2)$.

Results Compared to day workers, night shift workers had hypermethylation in the promoter region of CSNK1E $(q=0.15)$. Women who worked night shifts for $\geq 10$ years exhibited hypomethylation in the body of NR1D1 $(q=0.08)$ compared to those with $<10$ years of history. Hypermethylation in the body of ARNTL was also apparent for those who worked $\geq 3$ consecutive night shifts a week compared to $<3$ nights $(\mathrm{q}=0.18)$. Among night shift workers, melatonin patterns (24-hr concentrations, peak timing) were associated with methylation at three loci (RORA, MTNR1A, PER3) (q $\leq$ 0.20 ). No association between melatonin and methylation was identified among day workers.
Conclusion These findings suggest that circadian misalignment among night shift workers is associated with differential methylation in several circadian genes, but larger studies are needed to confirm.

\section{0-313 NIGHT SHIFT WORK, SLEEP DISORDERS AND LUNG CANCER RISK AMONG WOMEN: RESULTS FROM A POPULATION-BASED CASE-CONTROL STUDY IN FRANCE (THE WELCA STUDY)}

${ }^{1}$ Emilie Cordina-Duverger, Nastassia Tvardik, Diane Martin, Shreeshti Uchai, Regine Billmann, Pascal Guenel, Loredana Radoï. 'INSERM, France

\subsection{6/OEM-2021-EPI.142}

Introduction Night shift work may lead to sleep disorders and circadian rhythm disruption. It was classified as probably carcinogenic by IARC but, unlike breast or prostate cancer, there is only scant evidence of an association with lung cancer.

Objective To explore the role of night shift work and sleep disorders in lung cancer risk among women.

Methods Information on night work and sleep disorders over lifetime was obtained in a case-control study on female lung cancer in 716 cases and 758 population controls in the Paris area (WELCA). Logistic regression models adjusting for tobacco smoking and other relevant confounders were used to estimate odds ratios (OR) and 95\% confidence intervals (CI) associated with night work exposure metrics (years of night work, frequency of night shifts), sleep duration per day ( $<7 \mathrm{~h}, 7 \mathrm{~h}, \geq 8 \mathrm{~h}$ ) and sleep disorders (difficulty in falling asleep; waking up at night, too early or too tired; intake of sleep medicine). A sleep disruption index (SDI) was used to classify women by number of sleep disturbances in categories of low ( 0 or 1$)$, medium ( 2 or 3 ) or high SDI (4 or 5).

Results The OR was 1.08 (95\% CI 0.75-1.56) in women who ever worked at night. This OR did not increase with duration or frequency of night shifts. Women who slept 8 or more hours per day had an OR of 1.40 (95\% CI 1.04-1.87) as compared to those who slept 7 hours. Women who had both high SDI and who worked at night for 6 or more years had an OR of 3.61 (95\% CI: 1.27-10.23).

Conclusion Lung cancer risk among women was not associated with ever working at night in our study. The association with longer sleep duration is intriguing and warrants further scrutiny.

\section{0-384 THE ASSOCIATION BETWEEN NIGHT SHIFT WORK AND RISK OF BREAST CANCER: RESULTS FROM THE NIGHTINGALE STUDY}

${ }^{1}$ Nina Berentzen, Anouk Pijpe, Roel Vermeulen, Jelle Vlaanderen, Hans Kromhout, Flora van Leeuwen, Matti Rookus. 'Netherlands Cancer Institute, Netherlands

\subsection{6/OEM-2021-EPI.143}

Introduction and Objectives The International Agency for Research on Cancer confirmed night shift work as a 'probable' carcinogen in an updated evaluation (2019). Noting that evidence from human studies is still inconclusive, in particular due to the lack of prospective cohort studies with detailed exposure assessment. We prospectively examined different 
metrics of night work (e.g. duration, lifetime mean no. nights per month and cumulative no. nights) and risk of breast cancer, and whether the association was dependent on age or change in body weight.

Methods Overall, 55,350 female nurses completed extensive questions on shift work and were followed for incident breast cancer. Cox regression yielded multivariable-adjusted breast cancer incidence hazard ratios (HRs) and 95\% confidence intervals (CIs) for night work groups vs never night work, and Wald tests were used to assess potential interaction with age and change in body weight.

Results During 5,5 years of follow-up, 687 cases of incident invasive breast cancer were registered. The age adjusted HRs were 1.26 (95\% CI 1.01-1.57) for ever vs never night work, and 1.32 (95\% CI 1.01-1.74) for the tertile with the greatest cumulative no. nights ( $\geq 991$ nights) vs never night work. No trends were observed with increasing levels of different exposure metrics. There was a statistically significant interaction between night work and weight gain on risk of breast cancer; among women with $12-18 \mathrm{~kg}$ weight gain since age 18 , the HR for ever vs never night work was 2.45 (95\% CI 1.28 to 4.68).

Conclusion Higher duration, mean no. nights per month and cumulative no. nights were associated with a moderately increased risk of invasive breast cancer. Particularly for night shift workers with weight gain of $12-18 \mathrm{~kg}$, the risk was increased. Our results point towards a potential interaction between change in body weight and night shift work on breast cancer risk.

\section{Specific Occupations}

\section{0-57 ALUMINUM DUST EXPOSURE AND RISK OF NEURODEGENERATIVE DISEASES IN A COHORT OF MALE MINERS IN ONTARIO, CANADA}

'Xiaoke Zeng, Jill MacLeod, Colin Berriault, Nathan DeBono, Victoria H Arrandale, Anne M Harris, Paul Demers. 'University of Toronto, Canada

\subsection{6/OEM-2021-EPI.144}

Introduction McIntyre Powder (MP), a fine-sized aluminum and aluminum compound powder, was administered to Ontario miners from 1943 to 1979 as purported prophylaxis against silicosis. Aluminum has long been suspected of having a role in the development of neurological diseases. However, very few studies have examined the risk of neurological disease among miners with exposure to aluminum dust, and previous findings were inconclusive.

Objectives We estimated associations between respirable aluminum exposure through MP and neurological disease in a retrospective cohort of mining workers from Ontario, Canada. Outcomes included Alzheimer's disease, Alzheimer's with other dementias, Parkinson's disease, parkinsonism, and motor neuron disease.

Methods The cohort was created by linking a database of mining workers' work history to health care records. This analysis included 36,826 male miners potentially exposed to MP between 1943 and 1979, followed up for disease diagnosis between 1992 and 2018. Exposure was assessed using two approaches, self-reported and historical records. Neurological diseases were ascertained using physician billing and hospital discharge records. Poisson regression models were used to estimate associations between MP exposure and neurological outcomes using incidence rate ratios and 95\% confidence intervals (RR, 95\% CI).

Results Exposure to self-reported MP was associated with an elevated incidence rate of Parkinson's disease (RR 1.34, 95\% CI: 1.14, 1.57). The rate of Parkinson's disease appeared to increase with the duration of exposure assessed by historical records. Ever-exposure to MP was positively associated with an elevated rate of Alzheimer's with other dementias (RR 1.12, 95\% CI 1.06, 1.19), but not $\mathrm{Alz}$ heimer's disease alone.

Conclusion This study found that miners who were exposed to respirable aluminum, as McIntyre Powder, had elevated rates of Parkinson's disease. The rate of Parkinson's disease appeared to increase with the duration of exposure assessed by historical records.

\section{0-105 MATERNAL OCCUPATION AS A NAIL TECHNICIAN OR HAIRDRESSER DURING PREGNANCY AND BIRTH DEFECTS, NATIONAL BIRTH DEFECTS PREVENTION STUDY, 1997-2011}

${ }^{1}$ Miriam R Siegel, Carissa M Rocheleau, Kendra Broadwater, Albeliz Santiago-Colón, Michele L Herdt, I-Chen Chen, Christina C Lawson, Candice Johnson. ${ }^{1}$ CDC/NIOSH, United States

\subsection{6/OEM-2021-EPI.145}

Introduction Nail technicians and hairdressers may be exposed to products containing chemicals with potential reproductive effects. While studies have examined birth defects in children of cosmetologists and hairdressers, nail technician work has not been individually evaluated as a risk factor for birth defects.

Objectives We investigated associations between maternal occupation as a nail technician or hairdresser during pregnancy (versus non-cosmetologist) and selected birth defects.

Methods We analyzed population-based case-control data from the multisite National Birth Defects Prevention Study, 19972011. Cases were fetuses or infants with major structural birth defects; controls were liveborn infants without major birth defects. For 31,652 case and 11,613 control mothers, expert raters classified self-reported maternal jobs into discrete categories as nail technician, hairdresser, combination nail technician-hairdresser, other cosmetologist, or non-cosmetologist. We used logistic regression to calculate odds ratios (ORs) and 95\% confidence intervals (CIs) for associations between occupation during the first trimester of pregnancy and birth defect type, controlling for age, smoking, education, and race/ ethnicity.

Results Sixty-one mothers worked as nail technicians, 196 as hairdressers, 39 as combination nail technician-hairdressers, and 42,810 as non-cosmetologists during pregnancy. Strongest associations among nail technicians included multiple congenital heart defect (CHD) groups: any $\mathrm{CHD}(\mathrm{OR}=2.7$; $\mathrm{CI}: 1.3-$ 5.9); conotruncal $(\mathrm{OR}=3.0$; $\mathrm{CI}: 1.0-8.8)$ (Tetralogy of Fallot $[\mathrm{OR}=3.5 ; \mathrm{CI}: 1.0-12.9])$; right ventricular outflow tract obstruction $(\mathrm{OR}=3.2$; CI: $1.0-10.4)$; and septal $(\mathrm{OR}=3.1$; CI: 1.2-8.1). Cleft lip with cleft palate was associated with occupation as a hairdresser $(\mathrm{OR}=2.0 ; \mathrm{CI}$ : 1.1-3.7). All oral cleft groups were associated with combination nail technician-hairdresser work (ORs ranging from 4.2 to 5.3).

Conclusion Despite small samples, results suggest associations between maternal nail technician work during pregnancy and 